\title{
Digestive System Neuroendocrine Carcinoma
}

National Cancer Institute

\section{Source}

National Cancer Institute. Digestive System Neuroendocrine Carcinoma. NCI Thesaurus.

Code C95405.

A high grade poorly differentiated carcinoma arising from the digestive system. It is composed of small or large neoplastic cells that express immunohistochemical evidence of neuroendocrine differentiation. There is marked nuclear atypia, necrosis, and more than 20 mitoses per $10 \mathrm{HPF}$. 